\title{
Complex Dynamics of Glass-Forming Liquids
} A mode-coupling theory

\author{
WOLFGANG GÖTZE
}

Fakultät für Physik,

Technische Universität München 


\section{CONTENTS}

Preface V

1 Glassy dynamics of liquids-facets of the phenomenon 1

1.1 Stretching of the dynamics 1

1.2 Power-law relaxation 8

$\begin{array}{lll}1.3 & \text { Superposition principles } & 15\end{array}$

1.4 Two-step relaxation through a plateau $\quad 21$

1.5 The cage effect 24

1.6 Crossover phenomena 30

1.7 Hard-sphere systems: the paradigms 37

1.8 Hard-sphere systems with short-range attraction 44

2 Correlation functions 51

2.1 The evolution of dynamical variables 54

2.2 Correlation-function description of the dynamics 61

2.3 Spectral representations 66

2.4 Memory-kernel descriptions of correlators $\quad 72$

2.4.1 Zwanzig-Mori equations $\quad 72$

2.4.2 Models for correlation functions $\quad 78$

2.5 Linear-response theory $\quad 82$

2.6 The arrested parts of correlation functions $\quad 89$

3 Elements of liquid dynamics $\quad 96$

$\begin{array}{lll}3.1 & \text { Preliminaries } & 96\end{array}$

3.1.1 Homogeneous isotropic systems without chirality 96

3.1.2 Densities and density fluctuations $\quad 100$

$\begin{array}{ll}3.2 & \text { Tagged-particle dynamics } \\ & 107\end{array}$

3.2.1 Basic concepts and general equations 107

3.2.2 Tagged-particle diffusion $\quad 112$

$\begin{array}{ll}\text { 3.2.3 The friction coefficient } & 120\end{array}$

3.2.4 The cage effect and glassy-dynamics precursors

3.3 Densities and currents in simple liquids 132

3.3.1 Definitions and general equations 132

$\begin{array}{ll}\text { 3.3.2 Transverse-current diffusion } & 142\end{array}$

3.3.3 The generalized-hydrodynamics description of
transverse-current correlations

3.3.4 Visco-elastic features and glassy-dynamics precursors of the transverse-current correlators 
3.3.5 Representations of the density correlators in terms of relaxation kernels

3.3.6 Sound waves and heat diffusion

3.3.7 Visco-elastic features and glassy-dynamics precursors of the density-fluctuation correlators

4 Foundations of the mode-coupling theory for the evolution of glassy dynamics in liquids

4.1 Self-consistent-current-relaxation approaches 178

$\begin{array}{lll}\text { 4.1.1 The factorization ansatz } & 178\end{array}$

4.1.2 Self-consistency equations for density correlators $\quad 185$

$\begin{array}{ll}4.2 \text { A mode-coupling theory } & 191\end{array}$

4.2.1 Equations of motion and fixed-point equations 191

4.2.2 Mode-coupling-theory models 196

4.2.3 The basic version of microscopic mode-coupling theories

4.2.4 An elementary mode-coupling-theory model 209

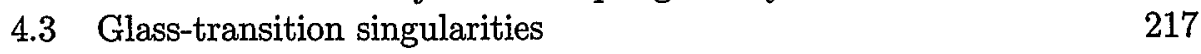

4.3.1 Regular and critical states 217

4.3.2 Examples for bifurcation diagrams $\quad 224$

4.3.3 Classification of the critical states 235

4.3.4 Correlation arrest near $A_{2}$ singularities $\quad 242$

4.3.5 Density-fluctuation arrest in hard-sphere-like systems 250

4.3.6 Arrest in systems with short-ranged-attraction 256

4.4 Dynamics near glass-transition singularities 267

4.4.1 Relaxation through plateaus 267

4.4.2 Below-plateau relaxation 281

4.4.3 Structure and structure relaxation $\quad 289$

4.4.4 Descriptions of some glassy-dynamics data 292

5 Extensions of the mode-coupling theory for the evolution of glassy dynamics of liquids 304

5.1 Extensions of the MCT for simple systems 305

5.1.1 MCT equations for the glassy shear dynamics 305

5.1.2 Glassy-relaxation features of shear correlations 308

5.1.3 MCT equations for the tagged-particle dynamics 320

5.1.4 Idealized transitions from diffusion to localization 331

5.1.5 Glassy-dynamics features of tagged-particle motions 345

5.2 A mode-coupling theory for mixtures of spherical particles $\quad 356$

5.2.1 The equations of motion $\quad 357$

5.2.2 Density-fluctuation arrest $\quad 366$

5.2.3 Hard-sphere mixtures 375

$\begin{array}{ll}\text { 5.2.4 Sodium-disilicate melts } & 383\end{array}$ 
5.3 A mode-coupling theory for molecular liquids 386

5.3.1 A theory for interaction-site-density correlators $\quad 387$

5.3.2 Systems of symmetric dumbbells $\quad 395$

5.3.3 Glassy Rouse dynamics 427

5.4 Some addenda 432

6 Asymptotic relaxation laws $\quad 437$

6.1 Dynamics of the first-scaling-law regime 438

6.1.1 Reformulation of the MCT equations of motion 438

6.1.2 The critical dynamics 443

6.1.3 Asymptotic description of the $A_{2}$-bifurcation dynamics $\quad 470$

6.1.4 The scaling-limit description of the generic liquid-glass-transition dynamics 492

6.1.5 Extended scaling-limit description of the generic $A_{2}$-bifurcation dynamics 502

6.2 Dynamics of the second-scaling-law regime 513

6.2.1 Equations of motion for the second-scaling-law regime $\quad 514$

6.2.2 The second-scaling-law description of the liquid dynamics 519

6.2.3 Asymptotic corrections for the second scaling limit 533

6.3 Relaxation near higher-order singularities 538

6.3.1 Correlation arrest near higher-order singularities $\quad 539$

6.3.2 Logarithmic relaxation 553

A Mathematical miscellanies $\quad 577$

$\begin{array}{lll}\text { A.1 Laplace transforms } & 577\end{array}$

A.2 Fourier transforms $\quad 580$

A.3 Positive-definite and positive-analytic functions 583

A.4 Harmonic-oscillator correlators $\quad 588$

$\begin{array}{ll}\text { A.5 Matrix correlators } & 591\end{array}$

A.6 Product correlators $\quad 594$

A.7 Power-law variations $\quad 596$

$\begin{array}{ll}\text { A.8 Logarithmic variations } & 601\end{array}$

B Symmetries of fluctuation correlators 603

$\begin{array}{ll}\text { C Smoothened correlators } & 608\end{array}$

D Theorems on MCT equations 611

$\begin{array}{ll}\text { D.1 Convergence of the approximant sequences } & 611\end{array}$

D.2 Completely monotonic approximants 613

D.3 The maximum-eigenvalue inequality 616

D.4 Further properties of stability matrices 618

Bibliography 621

$\begin{array}{ll}\text { Index } & 635\end{array}$ 